\title{
An outbreak of carbapenem-resistant OXA-48 - producing Klebsiella pneumonia associated to duodenoscopy
}

\author{
Axel Kola ${ }^{1 *}$, Brar Piening ${ }^{1}$, Ulrich-Frank Pape ${ }^{2}$, Wilfried Veltzke-Schlieker ${ }^{2}$, Martin Kaase ${ }^{3}$, Christine Geffers ${ }^{1}$, \\ Bertram Wiedenmann ${ }^{2}$ and Petra Gastmeier ${ }^{1}$
}

\begin{abstract}
Background: Carbapenemase-producing Enterobacteriaceae (CPE) have become a major problem for healthcare systems worldwide. While the first reports from European hospitals described the introduction of CPE from endemic countries, there is now a growing number of reports describing outbreaks of CPE in European hospitals. Here we report an outbreak of Carbapenem-resistant K. pneumoniae in a German University hospital which was in part associated to duodenoscopy.
\end{abstract}

Findings: Between December 6, 2012 and January 10, 2013, carbapenem-resistant K. pneumoniae (CRKP) was cultured from 12 patients staying on 4 different wards. The amplification of carbapenemase genes by multiplex PCR showed presence of the bla OXA-48 gene. Molecular typing confirmed the identity of all 12 isolates. Reviewing the medical records of CRKP cases revealed that there was a spatial relationship between 6 of the cases which were located on the same wards. The remaining 6 cases were all related to endoscopic retrograde cholangiopancreatography (ERCP) which was performed with the same duodenoscope. The outbreak ended after the endoscope was sent to the manufacturer for maintenance.

Conclusions: Though the outbreak strain was also disseminated to patients who did not undergo ERCP and environmental sources or medical personnel also contributed to the outbreak, the gut of colonized patients is the main source for CPE. Therefore, accurate and stringent reprocessing of endoscopic instruments is extremely important, which is especially true for more complex instruments like the duodenoscope (TJF Q180V series) involved in the outbreak described here.

Keywords: K. pneumoniae, Carbapenemases, OXA-48, Outbreak, Endoscopy

\section{Background}

Carbapenemase-producing Enterobacteriaceae (CPE) are spreading worldwide, thereby increasing the problem of antimicrobial resistance for clinical and public health [1]. In healthcare facilities, $\mathrm{CPE}$ can cause serious infections and hospital outbreaks [2]. There are a few outbreak reports describing the association with duodenoscopy, [3-5] such as the recent outbreak of New Delhi metallo-ß-lactamase (NDM)-producing Escherichia coli transmitted by

\footnotetext{
*Correspondence: axel.kola@charite.de

'Institute of Hygiene and Environmental Medicine, Charité - University Medicine Berlin, Berlin, Germany

Full list of author information is available at the end of the article
}

endoscopic retrograde cholangiopancreatography (ERCP) in Illinois, USA $[6,7]$.

Here we report an outbreak of Klebsiella pneumoniae producing the oxacillinase-48 (OXA-48) carbapenemase which took place in Charité University Medicine, Berlin, a tertiary hospital with 139.000 hospital admissions a year.

Between December 6, 2012 and January 10, 2013, carbapenem-resistant $K$. pneumoniae (CRKP) was cultured from 5 patients staying on ward A. The patients were immediately transferred to single rooms or cohorted in shared rooms. Contact isolation precautions were taken for all 5 patients. Active surveillance screening for CRKP was introduced for all patients admitted to ward A and 
environmental samples from surfaces, medical devices, drugs, water, cleaning solutions, disinfectants etc. were taken. Neither the active surveillance nor the environmental samples revealed any further positive results for CRKP on ward A.

It was 4 weeks after the last CRKP was detected on ward A, when another 5 cases of CRKP emerged on different locations of the hospital: On ward B, there were 3 patients and on wards $\mathrm{C}$ and $\mathrm{D}$ each one further patient with CRKP-positive results.

\section{Methods}

The medical records of patients with CRKP were reviewed. As four patients (one from ward A, one from ward B and the two single patients from wards $C$ and $D$ ) underwent duodenoscopy for ERCP, the endoscopy records were also inspected.

From December 10, 2012 (after the second patient had been tested positive for CRKP) Patients who were admitted to wards $\mathrm{A}$ and $\mathrm{B}$ or underwent duodenoscopy using one specific instrument (TJF Q180V series) were screened for rectal CRKP colonization. In addition, the environment and all duodenoscopes of the endoscopy unit were sampled.

CRKP screening of patients was performed by plating rectal swabs on selective culture media containing cefpodoxime (ChromID ESBL, bioMerieux) and on MacConkey plates on which an ertapenem disk $(10 \mu \mathrm{g})$ was placed.

Swabs taken for environmental sampling were enriched in trypticase soy broth (TSB) for 7 days at $37^{\circ} \mathrm{C}$. Liquid samples were filtered through $0.2 \mu \mathrm{m}$ cellulose membrane filters which were enriched in TSB for 7 days at $37^{\circ} \mathrm{C}$. Disinfectants and soaps $(1 \mathrm{~mL})$ were transferred to TSB containing neutralizers (3\% Tween 80 , 3\% saponin, $0.1 \%$ histidin, $0.1 \%$ cystein). Subsequently, the enriched TSB samples were cultured on Columbia and MacConkey plates.

Duodenoscopes were sampled by flushing each channel with $20 \mathrm{ml}$ of sterile saline solution and swabbing the ends of the channels. A $10 \mathrm{~mL}$ sample of the flushing solution was neutralized and filtered. Filters and swabs were processed as described above.

Species identification and susceptibility testing was done using a VITEK 2 system.

For detection of carbapenemase genes, a multiplex polymerase chain reaction (PCR) containing primers for $b l a_{\mathrm{OXA}-48}, b l a_{\mathrm{BIC}}, b l a_{\mathrm{NDM}}$ and $b l a_{\mathrm{KPC}}$ was performed [8].

Strain typing of CRKP was done by comparison of XbaI macro restriction profiles generated by pulsed field gel electrophoresis (PFGE) according to the criteria of Tenover [9].

\section{Results}

An overview of the spatial and temporal relationship and the characteristics of the patients is given in Figure 1 and Table 1 , respectively.

Reviewing the endoscopy records of the four CRKP positive patients who underwent ERCP revealed that the same duodenoscope had been used in all of them. The flushing solutions and swabs from the respective duodenoscope grew no additional CRKP, nor did the samples from the other duodenoscopes or the environment of the endoscopy unit. Only enterococci were cultured from the flushing solution of one of the other duodenoscopes.

The review of the endoscope records identified 22 additional patients who underwent ERCP with the respective duodenoscope, of which 19 were available for

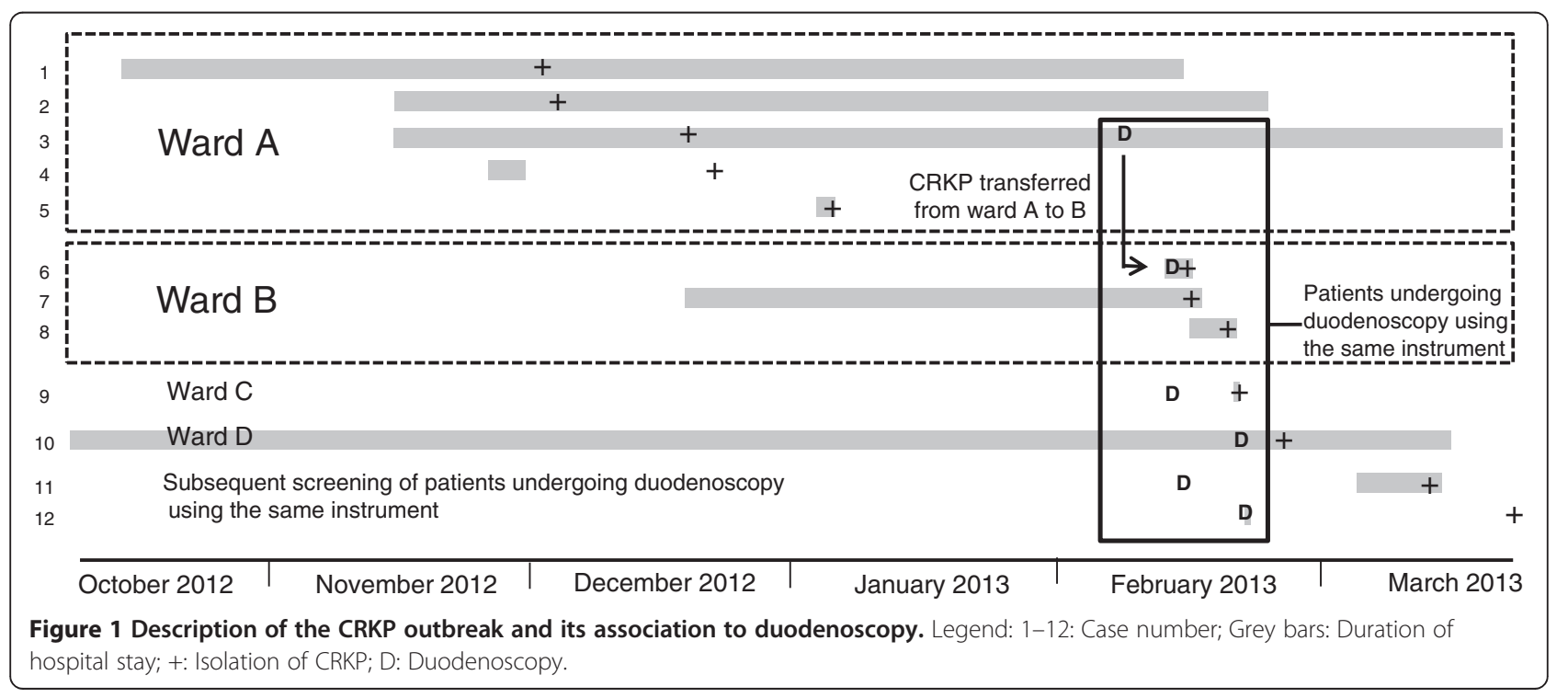


Table 1 Characteristics of CRKP cases

\begin{tabular}{llllllll}
\hline Patient & Gender & Age (years) & $\begin{array}{l}\text { Date of positive } \\
\text { CRKP sample }\end{array}$ & Source & Infection/colonization & Ward & Date of duodenoscopy \\
\hline 1 & $\mathrm{~m}$ & 71 & $06 / 12 / 12$ & Tracheobronchial secretion & Respiratory tract infection & $\mathrm{A}$ & - \\
2 & $\mathrm{~m}$ & 20 & $10 / 12 / 12$ & Rectal swab & Septicemia & $\mathrm{A}$ & - \\
3 & $\mathrm{~m}$ & 25 & $27 / 12 / 12$ & Rectal swab & Septicemia & $\mathrm{A}$ & $08 / 02 / 13$ \\
4 & $\mathrm{f}$ & 34 & $30 / 12 / 12$ & Blood culture & Septicemia & $\mathrm{A}$ & - \\
5 & $\mathrm{f}$ & 72 & $10 / 01 / 13$ & Rectal swab & Colonization & $\mathrm{A}$ & - \\
6 & $\mathrm{~m}$ & 58 & $13 / 02 / 13$ & Blood culture & Septicemia & $\mathrm{B}$ & $12 / 02 / 13$ \\
7 & $\mathrm{f}$ & 32 & $14 / 02 / 13$ & Intra-abdominal swab & Septicemia & $\mathrm{B}$ & - \\
8 & $\mathrm{~m}$ & 57 & $18 / 02 / 13$ & Rectal swab & Colonization & $\mathrm{B}$ & - \\
9 & $\mathrm{~m}$ & 26 & $18 / 02 / 13$ & Rectal swab & Surgical site infection & $\mathrm{C}$ & $12 / 02 / 13$ \\
10 & $\mathrm{~m}$ & 39 & $26 / 02 / 13$ & Rectal swab & Respiratory tract infection & $\mathrm{D}$ & $20 / 02 / 12$ \\
11 & $\mathrm{~m}$ & 43 & $14 / 03 / 13$ & Rectal swab & Septicemia & $\mathrm{E}$ & $13 / 02 / 13$ \\
12 & $\mathrm{f}$ & 61 & $24 / 03 / 13$ & Rectal swab & Colonization & $\mathrm{F}$ & $20 / 02 / 13$ \\
\hline
\end{tabular}

rectal screening. From two of these patients, CRKP were recovered from the rectal swabs.

Typing of the 12 CRKP strains (5 from ward A, 3 from ward $\mathrm{B}$ and the 4 single patients who underwent ERCP) revealed that they were closely related (less than 3 bands difference in PFGE). The amplification of carbapenemase genes by multiplex PCR showed presence of the bla $a_{\text {OXA- } 48}$ gene.

Apart from the ERCP procedure using the same duodenoscope, the review of the medical records did not develop any further linkage between the patients from wards $\mathrm{A}$ and $\mathrm{B}$ and the four single CRKP cases.

\section{Discussion}

In our hypothesis, CRKP were first introduced to ward A by patient 1 as part of the patient's gut flora and then transmitted to patients $2-5$ on ward A, most probably by contact (either by the hands of staff members or by contaminated medical devices or surfaces). Subsequently, one of these patients (patient 3) underwent ERCP, which led to contamination of the duodenoscope and the transmission of CRKP to patient 6, who acted as the source of transmission of CRKP to the patients 7 and 8 on ward B. The contaminated duodenoscope is also thought to have transmitted CRKP to four additional patients (patients 9 to 12), who all underwent ERCP using the same endoscope.

Although culturing of the duodenoscope did not recover CRKP, this does not exclude its association with CRKP transmission. While recommended for surveillance testing of endoscope reprocessing in the German guidelines, [10] flushing of the instrument channels with $\mathrm{NaCl}$ may not be sensitive enough to discover endoscope contamination [11] - particularly after the instrument was reprocessed several times before sampling such as in this case.
Additionally brushing the endoscope channels might have been the method of choice to prove contamination with the respective outbreak strain of CRKP [12], but the instrument was sent to the manufacturer for maintenance before it could be probed again.

Nevertheless, there are several epidemiological hints pointing to the duodenoscope as source of the CRKPtransmission: i) duodenoscopy using the instrument in question was the only epidemiological link between patients from different wards ii) following duodenoscopy, CRKP were only cultured if the implicated instrument was used iii) after the instrument in question was sent for maintenance (which revealed defects of the external layers and the distal cap of the respective duodenoscope), no further CRKP were cultured.

Reviewing the complete reprocessing operation in our endoscopy unit, we could not identify any deviations from the procedures recommended by the manufacturer of the duodenoscope. However, in one instance enterococci were cultured from one of the reprocessed duodenoscopes, which are indicative for insufficient cleaning and disinfection [10]. Therefore, the reprocessing procedures were not in every case sufficient, most probably due to the complex physical design of the endoscope's distal end which complicated reprocessing as additional manual steps had to be performed strictly complying with the manufacturer's advice: The distal cap of the duodenoscope was not removable and required accurate manual brushing and locking of the forceps elevator at $45^{\circ}$ before automated reprocessing could be done.

Recently, an outbreak of NDM-producing E. coli associated with ERCP which ended after the duodenoscopes were sterilized with ethylene oxide was published [6,7]. The authors concluded that ERCP-related transmission should be considered in case of an outbreak with CPE, as the complex design of duodenoscopes "might pose a 
particular challenge for cleaning and disinfection" [6] and makes cleaning difficult. This conclusion is supported by a recent review which identified gastrointestinal endoscopy to be a risk factor for infection and colonization with CPE [13]. The complex physical design of the endoscope's distal end could also have contributed to the outbreak described here, as it required strict adherence to the procedures recommended by the manufacturer. In a recent safety communication the U.S. Food And Drug Administration states that the transmission of microorganisms may even occur when manufacturer reprocessing instructions are followed correctly and, therefore, is continuing to evaluate information about documented and potential infections due to duodenoscopic instruments. In the meantime, patients should be informed about the potential risks of duodenoscopy including infections due to transmission [14].

Though the outbreak strain was also disseminated to patients who did not undergo ERCP and environmental sources or medical personnel contributed to the outbreak on wards $\mathrm{A}$ and $\mathrm{B}$, the gut of colonized patients is the main source for CPE. Therefore, accurate and stringent reprocessing of endoscopic instruments is extremely important to prevent the transmission of multidrug resistant organisms or blood borne viruses in particular, when more complex endoscopic instruments are involved as in the outbreak described here.

\footnotetext{
Abbreviations

CPE: Carbapenemase-producing Enterobacteriaceae; CRKP: Carbapenemresistant K. pneumonia; ERCP: Endoscopic retrograde cholangiopancreatography; NDM: New Delhi metallo-ß-lactamase; OXA-48: Oxacillinase-48; PCR: Polymerase chain reaction; PFGE: Pulsed field gel electrophoresis; TSB: Trypticase soy broth.
}

\section{Competing interests}

The authors declare that they have no competing interests.

\begin{abstract}
Authors' contributions
AK was responsible for the acquisition and analysis of microbiological data and drafted the manuscript, BP, UFP, WV and CG provided patient information and infection control measures, MK provided and analyzed PFGE data. All authors read, reviewed and provided feedback to the manuscript. All authors read and approved the final manuscript.
\end{abstract}

\section{Author details}

${ }^{1}$ Institute of Hygiene and Environmental Medicine, Charité - University Medicine Berlin, Berlin, Germany. ${ }^{2}$ Medical Department, Division of Hepatology and Gastroenterology, Charité - University Medicine Berlin, Berlin, Germany. ${ }^{3}$ National Reference Laboratory for multidrug-resistant Gram-negative bacteria, Department for Medical Microbiology, Ruhr-University Bochum, Bochum, Germany.

Received: 14 November 2014 Accepted: 13 March 2015

Published online: 25 March 2015

\section{References}

1. Schwaber MJ, Carmeli Y. Carbapenem-resistant Enterobacteriaceae: a potential threat. JAMA. 2008:300:2911-3.

2. Akova M, Daikos GL, Tzouvelekis L, Carmeli Y. Interventional strategies and current clinical experience with carbapenemase-producing Gram-negative bacteria. Clin Microbiol Infect. 2012;18:439-48.

3. Carbonne A, Thiolet JM, Fournier S, Fortineau N, Kassis-Chikhani N, Boytchev I, et al: Control of a multi-hospital outbreak of KPC-producing Klebsiella pneumoniae type 2 in France, September to October 2009. Euro Surveill. 2010; 15:pii: 19734.

4. Alrabaa SF, Nguyen P, Sanderson R, Baluch A, Sandin RL, Kelker D, et al. Early identification and control of carbapenemase-producing Klebsiella pneumoniae, originating from contaminated endoscopic equipment. Am J Infect Control. 2013:41:562-4.

5. Naas T, Cuzon G, Babics A, Fortineau N, Boytchev I, Gayral F, et al. Endoscopy-associated transmission of carbapenem-resistant Klebsiella pneumoniae producing KPC-2 beta-lactamase. J Antimicrob Chemother. 2010;65:1305-6.

6. Anonymus. Notes from the field: New Delhi metallo-beta-lactamaseproducing Escherichia coli associated with endoscopic retrograde cholangiopancreatography - Illinois, 2013. MMWR Morb Mortal Wkly Rep. 2014;62:1051.

7. Epstein L, Hunter JC, Arwady MA, Tsai V, Stein L, Gribogiannis M, et al. New Delhi metallo-beta-lactamase-producing carbapenem-resistant Escherichia coli associated with exposure to duodenoscopes. JAMA. 2014;312:1447-55.

8. Poirel L, Walsh TR, Cuvillier V, Nordmann P. Multiplex PCR for detection of acquired carbapenemase genes. Diagn Microbiol Infect Dis. 2011;70:119-23.

9. Tenover FC, Arbeit RD, Goering RV, Mickelsen PA, Murray BE, Persing DH, et al. Interpreting chromosomal DNA restriction patterns produced by pulsed-field gel electrophoresis: criteria for bacterial strain typing. J Clin Microbiol. 1995;33:2233-9.

10. Anonymus. Hygiene requirements for the reprocessing of medical devices. Recommendation of the Commission for Hospital Hygiene and Infection Prevention (KRINKO) at the Robert Koch Institute (RKI) and the Federal Institute for Drugs and Medical Devices (BfArM). Bundesgesundheitsblatt Gesundheitsforschung Gesundheitsschutz. 2012;55:1244-310.

11. Aumeran C, Poincloux L, Souweine B, Robin F, Laurichesse $H$, Baud O, et al. Multidrug-resistant Klebsiella pneumoniae outbreak after endoscopic retrograde cholangiopancreatography. Endoscopy. 2010;42:895-9.

12. Kovaleva J, Peters FT, van der Mei HC, Degener JE. Transmission of infection by flexible gastrointestinal endoscopy and bronchoscopy. Clin Microbiol Rev. 2013;26:231-54.

13. Muscarella LF. Risk of transmission of carbapenem-resistant Enterobacteriaceae and related "superbugs" during gastrointestinal endoscopy. World J Gastrointest Endosc. 2014;6:457-74.

14. Design of Endoscopic Retrograde Cholangiopancreatography (ERCP). Duodenoscopes may impede effective cleaning: FDA safety communication, issued February 19, 2015. http://www.fda.gov/MedicalDevices/Safety/ AlertsandNotices/ucm434871.htm.

\section{Submit your next manuscript to BioMed Central and take full advantage of:}

- Convenient online submission

- Thorough peer review

- No space constraints or color figure charges

- Immediate publication on acceptance

- Inclusion in PubMed, CAS, Scopus and Google Scholar

- Research which is freely available for redistribution 